\title{
Atrial fibrillation alters the microRNA expression profiles of the left atria of patients with mitral stenosis
}

Hai Liu ${ }^{1 \dagger}$, Guang-xian Chen ${ }^{1 \dagger}$, Meng-ya Liang ${ }^{1}$, Han Qin ${ }^{1}$, Jian Rong ${ }^{2}$, Jian-ping Yao ${ }^{1}$ and Zhong-kai Wu ${ }^{1 *}$

\begin{abstract}
Background: Structural changes of the left and right atria associated with atrial fibrillation (AF) in mitral stenosis (MS) patients are well known, and alterations in microRNA (miRNA) expression profiles of the right atria have also been investigated. However, miRNA changes in the left atria still require delineation. This study evaluated alterations in miRNA expression profiles of left atrial tissues from MS patients with AF relative to those with normal sinus rhythm (NSR).
\end{abstract}

Methods: Sample tissues from left atrial appendages were obtained from 12 MS patients ( 6 with AF) during mitral valve replacement surgery. From these tissues, miRNA expression profiles were created and analyzed using a human miRNA microarray. Results were validated via reverse-transcription and quantitative PCR for 5 selected miRNAs. Potential miRNA targets were predicted and their functions and potential pathways analyzed via the miRFocus database.

Results: The expression levels of 22 miRNAs differed between the AF and NSR groups. Relative to NSR patients, in those with AF the expression levels of 45\% (10/22) of these miRNAs were significantly higher, while those of the balance $(55 \%, 12 / 22)$ were significantly lower. Potential miRNA targets and molecular pathways were identified.

Conclusions: AF alters the miRNA expression profiles of the left atria of MS patients. These findings may be useful for the biological understanding of AF in MS patients.

Keywords: Atrial fibrillation, Microrna, Mitral stenosis, Microarray, Mirfocus

\section{Background}

Atrial fibrillation (AF) is characterized as an irregular and sometimes rapid heart rate, with symptoms that include palpitations and shortness of breath. AF is the most common cardiac arrhythmia observed in clinical practice and constitutes a risk factor for ischemic stroke [1]. Despite recent significant advances in the understanding of the mechanisms associated with AF, complexities in the etiology of atrial electrical dysfunction (including a genetic component [2]) and the subsequent associated arrhythmia have prevented definitive elucidation [3].

\footnotetext{
* Correspondence: wuzhk@mail.sysu.edu.cn

${ }^{\dagger}$ Equal contributors

'Second Department of Cardiac Surgery, First Affiliated Hospital of Sun Yat-Sen University, 58 Zhongshan II Road, Guangzhou 510080, China Full list of author information is available at the end of the article
}

The progression from acute to persistent and then chronic AF is accompanied by changes in gene expression that lead to differences in protein expression and activity. MicroRNAs (miRNAs) are regulators of gene expression at the post-transcriptional level [4], and appear to have regulatory roles that underlie the pathophysiology of AF. Many studies have shown that miRNAs regulate key genetic functions in cardiovascular biology and are crucial to the pathogenesis of cardiac diseases such as cardiac development [5], hypertrophy/ heart failure [6], remodeling [7], acute myocardial infarction [8], and myocardial ischemia-reperfusion injury [9]. Currently, there is a growing body of literature that indicates that many miRNAs are involved in AF through their target genes $[7,10,11]$. 
AF can be an isolated condition, but it often occurs concomitantly with other cardiovascular diseases such as hypertension, congestive heart failure, coronary artery disease, and valvular heart disease [12]. AF is also prevalent in mitral stenosis (MS; a consequence of rheumatic fever), affecting approximately $40 \%$ of all MS patients [13]. MS is among the major cardiovascular diseases in developing countries where rheumatic fever is less well controlled, and $50 \%$ or more of patients with severe MS have AF. Patients with both AF and MS have a 17.5-fold greater risk of stroke and a four-fold higher incidence of embolism compared with people with normal sinus rhythm (NSR) [14,15].

Structural changes of the left atria (LA) and right atria (RA) associated with AF in MS patients are well established [13,14]. Recently, reports suggest that AF also alters the miRNA expression profiles in RA of MS patients $[16,17]$. However, miRNA changes in LA from MS patients with AF are still unknown. Given the complexity of the pathophysiology that may be associated with $\mathrm{AF}$, we need a better understanding of the miRNA changes in the LA, which may help in designing and developing new therapeutic interventions. This study investigated alterations of miRNA expression profiles in LA tissues of MS patients with AF relative to MS patients with NSR.

\section{Methods}

The Human Ethics Committee of First Affiliated Hospital of Sun Yat-sen University approved this study, and the investigation complied with the principles that govern the use of human tissues outlined in the Declaration of Helsinki. All patients gave informed consent before participating in the study.

\section{Human tissue preparation}

Left atrial appendage (LAA) tissue samples were obtained from MS patients, both in NSR ( $n=6$, without history of $\mathrm{AF}$ ) and with $\mathrm{AF}(\mathrm{n}=6$, documented arrhythmia $>6$ months before surgery). The tissue samples were obtained at the time of mitral valve surgery, immediately snap frozen in liquid nitrogen, and stored at $-80^{\circ} \mathrm{C}$ until used. The diagnosis of AF was reached by evaluating medical records and 12-lead electrocardiogram findings. NSR patients had no history of using antiarrhythmic drugs and were screened to ensure that they had never experienced AF [18]. Preoperative 2-dimensional color transthoracic echocardiography was performed routinely on the patients. Preoperative functional status was recorded according to New York Heart Association (NYHA) classifications.

\section{RNA isolation}

The total RNA from human LAA tissue samples was extracted using TRIzol reagent (Invitrogen) in accordance with the protocol of the manufacturer. The RNA quality of each sample was determined using an Agilent 2100 Bioanalyzer (Agilent Technologies, Santa Clara, CA, USA) and immediately stored at $-80^{\circ} \mathrm{C}$.

\section{Microarray processing and analysis}

The miRNA microarray expression analysis was performed by LC Sciences (Houston, TX, USA) as described previously [19]. In brief, the assay began with a total RNA sample (2 to $5 \mu \mathrm{g}$ ). The total RNA was sizefractionated using a YM-100 Microcon centrifugal filter (Millipore, Billerica, MA). RNA sequences of $<300 \mathrm{nt}$ were isolated. These small RNAs were then extended at the 3 ' end with a poly(A) tail using poly(A) polymerase, and then by ligation of an oligonucleotide tag to the poly(A) tail for later fluorescent dye staining.

Hybridization was performed overnight on a $\mu$ Paraflo microfluidic chip using a micro-circulation pump (Atactic Technologies, Houston, TX). Each microfluidic chip contained detection probes and control probes. The detection probes were made in situ by photogenerated reagents. These probes consisted chemically of modified nucleotide coding sequences complementary to target miRNA (all 1921 human miRNAs listed in the Sanger's miRNA miRBase, Release 18.0, http://microrna.sanger. ac.uk/sequences/) and a spacer segment of polyethylene glycol to extend the coding sequences away from the substrate. The hybridization melting temperatures were balanced by chemical modifications of the detection probes. Hybridization was performed using $100 \mu \mathrm{L}$ of $6 \times$ saline-sodium phosphate-EDTA (SSPE) buffer $(0.90 \mathrm{M}$ $\mathrm{NaCl}, 60 \mathrm{mM} \mathrm{Na}{ }_{2} \mathrm{HPO}$, 6 mM EDTA, pH 6.8) containing $25 \%$ formamide at $34^{\circ} \mathrm{C}$.

Fluorescence labeling with tag-specific Cy5 dye was used for after-hybridization detection. An Axon GenePix 4000B Microarray Scanner (Molecular Device, Union City, CA) was used to collect the fluorescent images, which were digitized using Array-Pro image analysis software (Media Cybernetics, Bethesda, MD). Each miRNA was analyzed two times and the controls were repeated 4-16 times.

Analysis of the microarray data was also performed at LC Sciences (see Additional file 1). The microarray data was analyzed by subtracting the background, and then the signals were normalized using a locally weighted regression scatterplot smoothing (LOWESS) filter as reported previously [20]. Detectable miRNAs were selected based on the following criteria: signal intensity $>3$-fold the background standard deviation, and spot coefficient of variation $(\mathrm{CV})<0.5$, where $\mathrm{CV}=$ standard deviation/ signal intensity. When repeating probes were present on the array, the transcript was listed as detectable only if the signals from at least $50 \%$ of the repeating probes were above detection level. To identify miRNAs whose 
expression differed between the AF and NSR groups, statistical analysis was performed. The ratio of two samples was calculated and expressed in $\log _{2}$ scale (balanced) for each miRNA. The miRNAs were then sorted according to their differential ratios. The $P$-values of the $t$-test were also calculated. miRNAs with $P$-values $<0.05$ were considered significantly differentially expressed.

\section{Reverse transcription-real time quantitative PCR (RT-qPCR) validation of selected miRNAs}

To validate the microarray results in the present study, a stem-loop RT-qPCR based on SYBR Green I was performed on selected differentially expressed miRNAs. The primers used are listed in Additional file 2. Total RNA was isolated using TRIzol reagent (Invitrogen) as described above. A single-stranded cDNA for each specific miRNA was generated by reverse transcription (RT) of $250 \mathrm{ng}$ of total RNA using a miRNA-specific stemlooped RT primer. Briefly, an RT reaction mixture contained $250 \mathrm{ng}$ of total RNA, $0.5 \mu \mathrm{L}$ of $2 \mu \mathrm{M}$ stem-loop RT primer, $1.0 \mu \mathrm{L}$ of $5 \times$ RT buffer, $0.25 \mu \mathrm{L}$ of $10 \mathrm{mM}$ of each dNTP, $0.25 \mu \mathrm{L}$ of $40 \mathrm{U} / \mu \mathrm{L}$ RNase inhibitor, and $0.5 \mu \mathrm{L}$ of $200 \mathrm{U} / \mu \mathrm{L}$ Moloney murine leukemia virus (MMLV) reverse transcriptase. An Eppendorf Mastercycler (Eppendorf, Hamburg, Germany) was used to perform the $\mathrm{RT}$ reaction under the following conditions: $42^{\circ} \mathrm{C}$ for $60 \mathrm{~min}, 70^{\circ} \mathrm{C}$ for $15 \mathrm{~min}$, and finally, held at $4^{\circ} \mathrm{C}$.

After the RT reaction, qPCR was performed using an ABI PRISM 7900HT sequence-detection system (Applied Biosystems, Foster City, CA, USA) with the Platinum SYBR Green qPCR SuperMix-UDG (Invitrogen). In accordance with the manufacturer's instructions, a $20-\mu \mathrm{L}$ PCR reaction mixture contained $0.5 \mu \mathrm{L}$ of $\mathrm{RT}$ product, $10 \mu \mathrm{L}$ of $2 \times$ SYBR Green Mix, $0.4 \mu \mathrm{L}$ of ROX, $0.8 \mu \mathrm{L}$ of $10 \mu \mathrm{M}$ primer mix, and $8.3 \mu \mathrm{L}$ of nuclease-free water. The reaction protocol was: $95^{\circ} \mathrm{C}$ for $2 \mathrm{~min}$, and then 40 amplification cycles of $95^{\circ} \mathrm{C}$ for $15 \mathrm{~s}$, and $60^{\circ} \mathrm{C}$ for $30 \mathrm{~s}$.

All reactions were run in triplicate. To account for possible differences in the amount of starting RNA, miRNA expressions were normalized to small nuclear RNA RNU6B $[21,22]$. RT-qPCR data were represented by the cycle threshold $(\mathrm{Ct})$ value. The relative expression level (i.e., fold change) for each miRNA was calculated using the comparative cycle threshold $2^{-\Delta \Delta \mathrm{Ct}}$ method [19].

\section{Target prediction and function analysis}

We used the database miRFocus (http://mirfocus.org/) to predict potential human miRNA target genes. The website describes miRFocus as a human miRNA information database, and is an open-source web tool developed for rapid analysis of miRNAs. It also provides comprehensive information concerning human miRNAs, including not only miRNA annotations but also miRNA and target gene interactions, correlations between miRNAs and diseases and signaling pathways, and more. The miRFocus provides a full gene description and functional analysis for each target gene by combining the predicted target genes from other databases (TargetScan, miRanda, PicTar, MirTarget and microT). In this study, only those genes that were predicted by two or more databases were considered candidates; the greater the number of databases that predicted that a given gene would be a target, the more likely the miRNA-mRNA interaction would be relevant [23]. The miRFocus program also identifies miRNA-enriched pathways, incorporating those from the Kyoto Encyclopedia of Genes and Genomes (KEGG), Biocarta, and Gene Ontology (GO) databases, with Fisher's exact test.

\section{Statistical analyses}

All data are presented as mean \pm standard deviation and analyzed with the paired $t$-test. Spearman's correlation coefficients were used to examine the association between validated miRNAs and left atrial size. $P<0.05$ was considered statistically significant.

\section{Results}

\section{Clinical characteristics of the NSR and AF patients}

There were no significant differences in terms of age, gender, or NYHA functional classification between the NSR and AF groups. Preoperative color Doppler echocardiography showed that the size of the left atria of AF patients was significantly greater than that of NSR patients as previously reported [24], while there were no differences in the left ventricular end-diastolic diameter or ejection fraction between the two groups of patients (Table 1).

miRNA expression profiles of LAA tissue from MS patients with and without $A F$

Of the 1898 human miRNAs analyzed, a total 213 miRNAs were detected; NSR patients expressed 155 miRNAs, while the AF patients expressed 208 miRNAs (Figure 1A). Among these, 150 miRNAs were common to the patients of both groups. 5 miRNAs were detected only in those with

Table 1 Clinical characteristics of the NSR and AF patients ( $n=6$, each)

\begin{tabular}{lcc}
\hline & NSR $(\mathbf{n}=\mathbf{6})$ & AF $(\mathbf{n}=\mathbf{6})$ \\
\hline Age (years) & $47.51 \pm 8.36$ & $49.42 \pm 11.87$ \\
Gender (male/female) & $3 / 3$ & $2 / 4$ \\
LA size (mm) & $38.31 \pm 5.32$ & $56.35 \pm 6.08^{*}$ \\
LVEDD (mm) & $42.35 \pm 4.43$ & $44.13 \pm 5.17$ \\
LVEF (\%) & $62.54 \pm 5.16$ & $60.73 \pm 4.04$ \\
NYHA classification & $\|(3 / 6) / I I(3 / 6)$ & $\|(2 / 6) / I I(4 / 6)$ \\
\hline
\end{tabular}

${ }^{*} P<0.05$ with respect to NSR patients. 
NSR, and 58 miRNAs were detected only in those with AF (Figure 1B).

However, the expression levels of most of the detected miRNAs were low, which is evident by their low signal intensities (less than 500 units). Of the 155 miRNAs detected in the NSR group, 73 emitted signals $<500$ units, while only 16 were $>5000$ units. On the other hand, of the 208 miRNAs detected in patients with AF, the signal intensities of 127 were $<500$ units, while only 16 were $>5000$ units (Figure 2). The signal intensities of the 5 miRNAs detected only in NSR, and the 58 miRNAs detected only in AF, were all $<200$ units and not high enough to consider them as differentially expressed between NSR and AF. Hence these were not considered here for further analysis.

Differences in miRNA expression profiles of LAA tissues from MS patients with and without AF

Differences existed in the expression levels of the 150 miRNAs detected in both the NSR and AF groups (Table 2). Statistical analysis showed that 22 of these miRNAs (15\%) were significantly dysregulated in the AF group relative to the NSR: 10 miRNAs (45\%) were upregulated, while $12(55 \%)$ were downregulated $(P<0.05)$.

Most of the miRNAs selected for further analysis via RT-qPCR had a fold change that satisfied the equation $\left|\log _{2}^{\text {(fold change) }}\right| \geq 1.5$ (Figure 3 ), and at least one group had a signal intensity $>2000$ units (Figure 4). Although

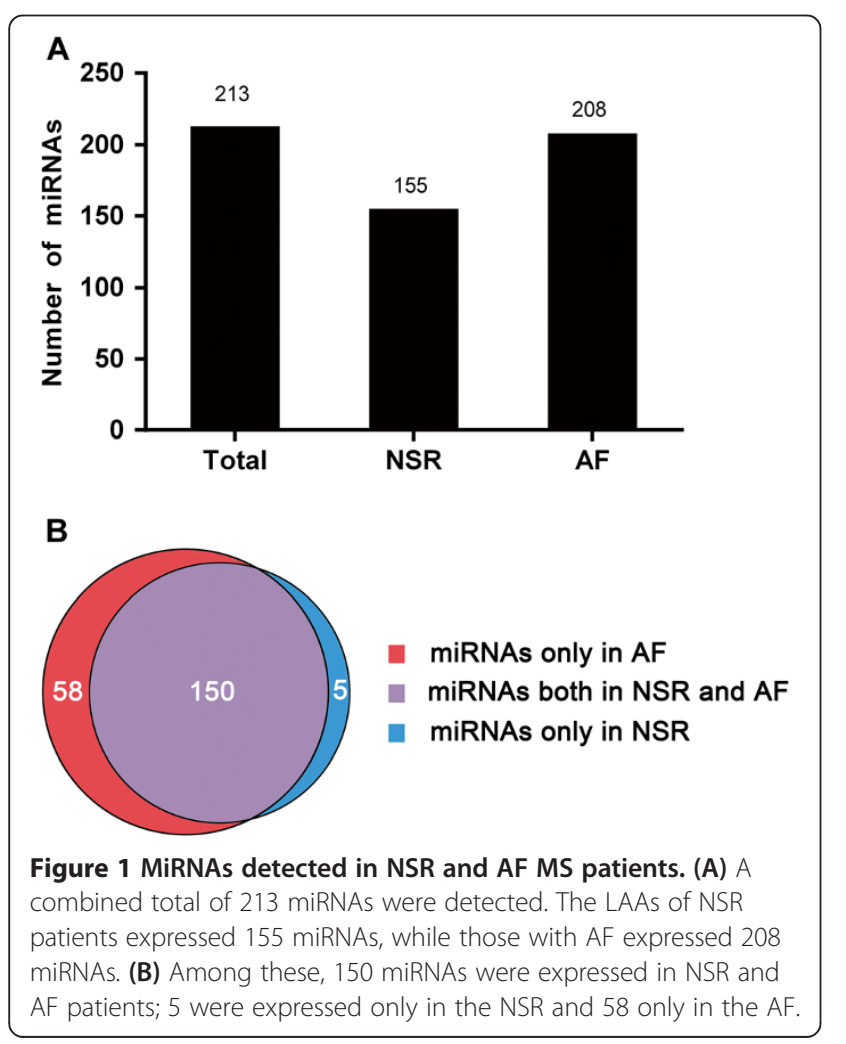

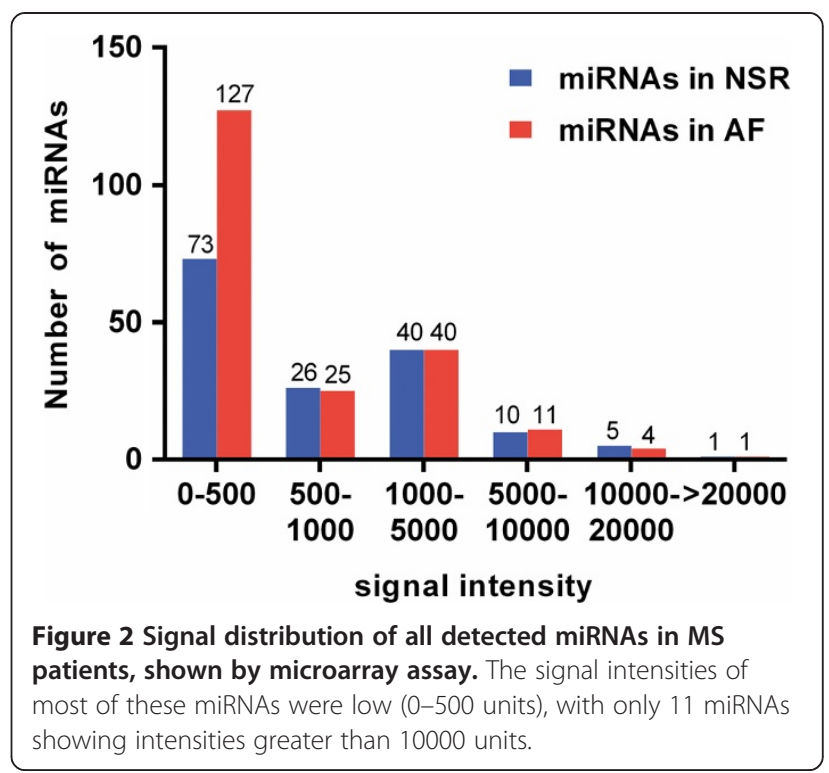

Table 2 MiRNAs differentially expressed in LAA tissues between MS patients with AF or NSR

\begin{tabular}{|c|c|c|c|c|}
\hline miRNAs & NSR signal & AF signal & $\log _{2}(\mathrm{AF} / \mathrm{NSR})$ & $P$-value \\
\hline \multicolumn{5}{|l|}{ Upregulated* } \\
\hline hsa-miR-3613-3p & 294.01 & 3062.72 & 3.38 & 0.011 \\
\hline hsa-miR-3196 & 1383.37 & 2477.49 & 0.84 & 0.013 \\
\hline hsa-miR-3178 & 185.46 & 374.05 & 1.01 & 0.020 \\
\hline hsa-miR-466 & 1183.25 & 3342.81 & 1.50 & 0.023 \\
\hline hsa-miR-574-3p & 4477.78 & 13515.75 & 1.59 & 0.024 \\
\hline hsa-miR-4492 & 334.39 & 834.17 & 1.32 & 0.027 \\
\hline hsa-miR-4707-5p & 386.91 & 857.24 & 1.15 & 0.029 \\
\hline hsa-miR-15b-5p & 382.60 & 723.26 & 0.92 & 0.030 \\
\hline hsa-miR-21-5p & 442.29 & 819.40 & 0.89 & 0.047 \\
\hline hsa-miR-4497 & 4574.24 & 6545.73 & 0.52 & 0.047 \\
\hline \multicolumn{5}{|l|}{ Downregulated* } \\
\hline hsa-miR-26a-5p & 9701.87 & 4313.64 & -1.17 & 0.007 \\
\hline hsa-miR-1 & 12740.13 & 3079.47 & -2.05 & 0.019 \\
\hline hsa-miR-195-5p & 611.70 & 384.70 & -0.67 & 0.022 \\
\hline hsa-miR-26b-5p & 794.41 & 198.33 & -2.00 & 0.023 \\
\hline hsa-miR-5100 & 775.16 & 362.75 & -1.10 & 0.029 \\
\hline hsa-miR-29a-3p & 589.33 & 512.76 & -0.20 & 0.029 \\
\hline hsa-miR-24-3p & 3269.76 & 2405.77 & -0.44 & 0.031 \\
\hline hsa-miR-361-5p & 652.46 & 259.00 & -1.33 & 0.035 \\
\hline hsa-miR-151a-5p & 693.52 & 302.25 & -1.20 & 0.039 \\
\hline hsa-miR-4454 & 1262.00 & 488.07 & -1.37 & 0.039 \\
\hline hsa-miR-720 & 951.72 & 618.64 & -0.62 & 0.047 \\
\hline hsa-let-7 g-5p & 4801.17 & 3524.47 & -0.45 & 0.047 \\
\hline
\end{tabular}

*Expression levels of miRNAs are described as upregulated or downregulated in AF relative to those in NSR. 


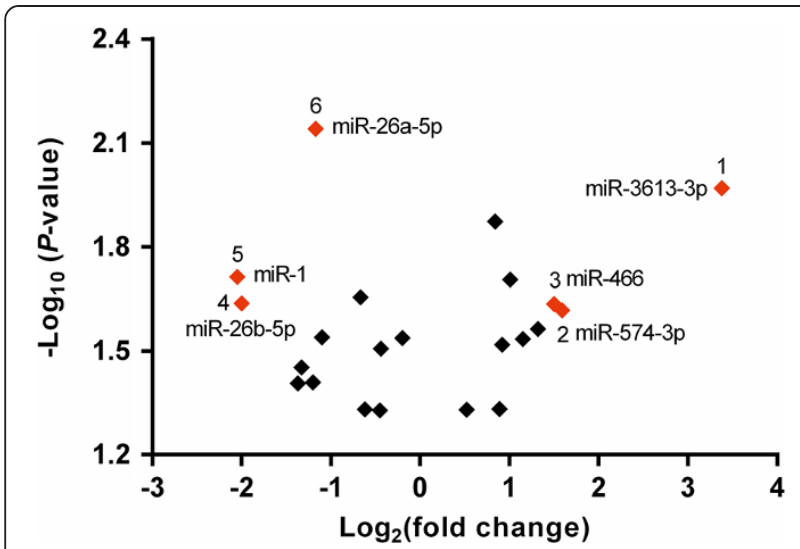

Figure 3 Volcano plot of 22 miRNAs significantly dysregulated in LAA tissues. The volcano plot shows the distribution of these miRNAs according to their $P$-value versus fold change. Those with the highest fold change in their expression $\left(\left|\log _{2}^{\text {(fold change) }}\right| \geq 1.5\right)$ have been labeled in red. Although the fold change of miR-26a-5p $\left(\left|\log _{2}^{\text {(fold change) }}\right|=1.17\right)$ does not meet the criteria, its $P$-value is $<0.01$ (i.e., $-\log _{10}^{(P \text {-value) }}>2$ ), and therefore is also labeled red.

the fold change of hsa-miR-26a-5p did not meet this criteria ( $\left|\log _{2}^{\text {(fold change) }}\right|=1.17$ ), its $P$-value was $<0.01$, and thus it was included in our selection. Finally, we selected 5 miRNAs for further analysis: 3 were upregulated in the AF group relative to the NSR (hsa-miR-466, hsa-miR574-3p, and hsa-miR-3613-3p), and 2 were downregulated (hsa-miR-1 and hsa-miR-26a-5p).

\section{Validation of the miRNA microarray data with RT-qPCR}

To validate the data obtained from the miRNA microarray, RT-qPCR was performed on 5 selected miRNAs, and the results were compared with the microarray (Figure 5, Additional file 3). According to the RT-qPCR data, hsa-miR-466, hsa-miR-574-3p, and hsa-miR-3613$3 p$ were upregulated in the LAAs of the AF group relative to the NSR, while hsa-miR-1 and hsa-miR-26a-5p were downregulated. These data are comparable with our microarray data and thus validate the results from the miRNA microarray for these miRNAs.

\section{Association between LA size and changes in miRNA expression}

We investigated whether the changes observed in the expression levels of the 5 validated miRNAs between the LAA tissues of NSR and AF patients correlated with LA size.

Spearman's correlation analysis showed a positive correlation between the level of expression of miR-466 in LAA and LA size $(r=0.73 ; P=0.007)$. Moreover, there was a significantly negative correlation between the levels of expression of miR-1 and miR-26a-5p in LAAs and LA size $(r=-0.81 ; P=0.002$ and $r=-0.86 ; P<0.001$, respectively). However, although the expression levels of

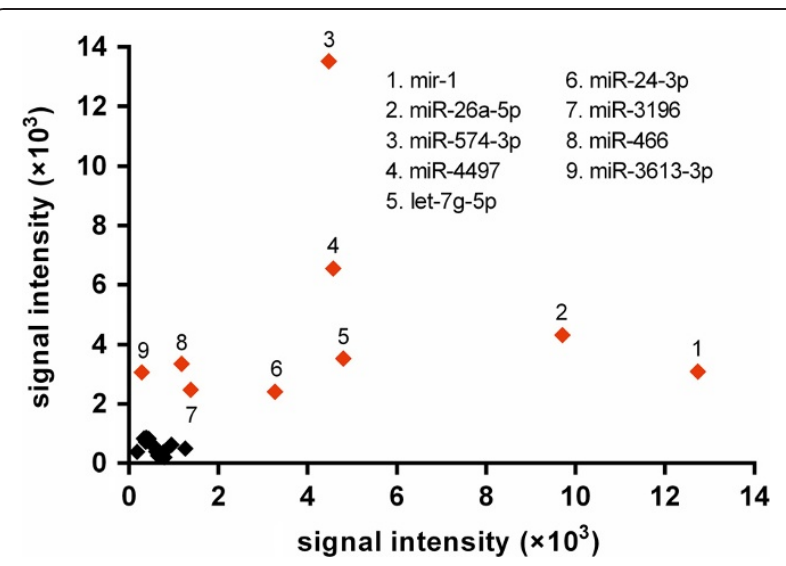

Figure 4 Comparison of signal intensities of 22 miRNAs significantly dysregulated in LAA tissues. The signal intensities of miRNAs expressed in the NSR group ( $x$-axis) and AF group (y-axis) were compared. The miRNAs with the higher signal intensities (>2000 units) either on the $x$-axis or $y$-axis were labeled red.

miR-574-3p and miR-3613-3p in LAAs significantly differed between NSR and AF patients, they were not significantly correlated with LA size $(r=0.54 ; P=0.07$ and $r=0.56 ; P=0.06$, respectively; Figure 6).

\section{Prediction of putative target genes and pathways of the differentially expressed miRNAs}

To determine the probable biological function of the differentially expressed miRNAs, we predicted the putative targets and pathways of 5 validated miRNAs (hsa-miR-1, hsa-miR-26a-5p, hsa-miR-466, hsa-miR-574-3p, and hsamiR-3613-3p) using the miRFocus database.

Numerous putative target genes and pathways were identified for the 5 miRNAs. The miRNAs hsa-miR-1 and hsa-miR-26a-5p were predicted by 5 target prediction databases; hsa-miR-574-3p was predicted from 4 target prediction databases, and hsa-miR-466 and hsamiR-3613-3P were predicted from 2 target prediction databases (Table 3).

The biological function and potential functional pathways of each putative gene target were classified using the GO term and KEGG pathway (Tables 4 and 5). Since every gene is associated with many GO terms and KEGG pathways, the significant GO term and KEGG pathway for each miRNA were identified with Fisher's exact test. Results of the target analysis indicated that the predicted potential genes have been linked to such important biological processes as the regulation of protein metabolism, transcription factor activity, cell division, and the transforming growth factor beta receptor (TGFBR) signaling pathway. These results suggest that these miRNAs have roles in human health and disease regulation. The pathway analysis also suggested that these miRNAs 


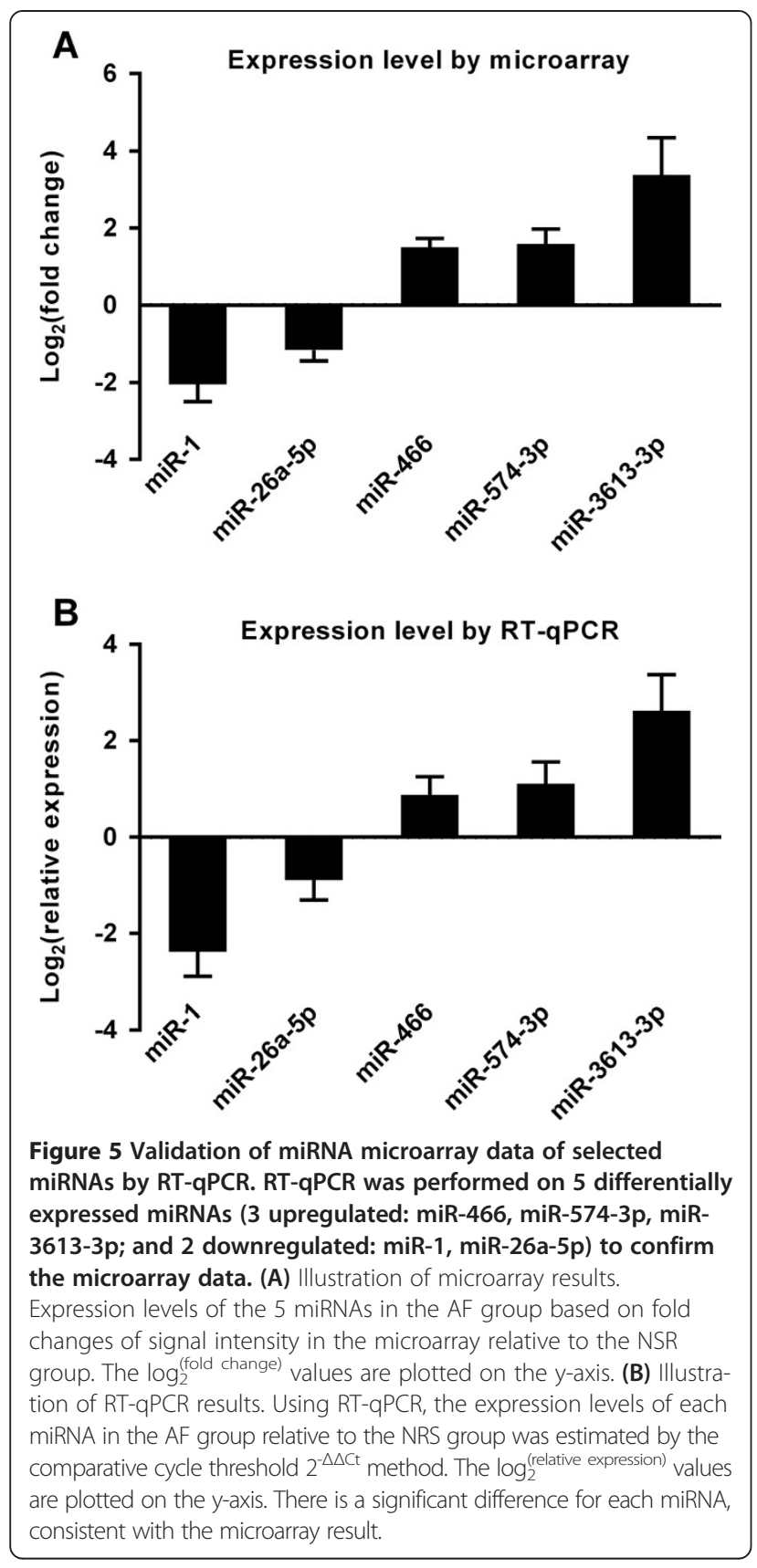

have important regulatory roles in different biological processes.

\section{Discussion}

More and more studies indicate that specific alterations in miRNA expression profiles are associated with specific disease pathophysiologies $[8,9,19]$. Xiao et al. [16] were the first to report miRNA alterations in the RA associated with AF in MS patients; 28 miRNAs were differentially expressed between MS patients with AF and those in NSR. However, miRNA changes due to AF in the LA of MS patients are still unknown. The present
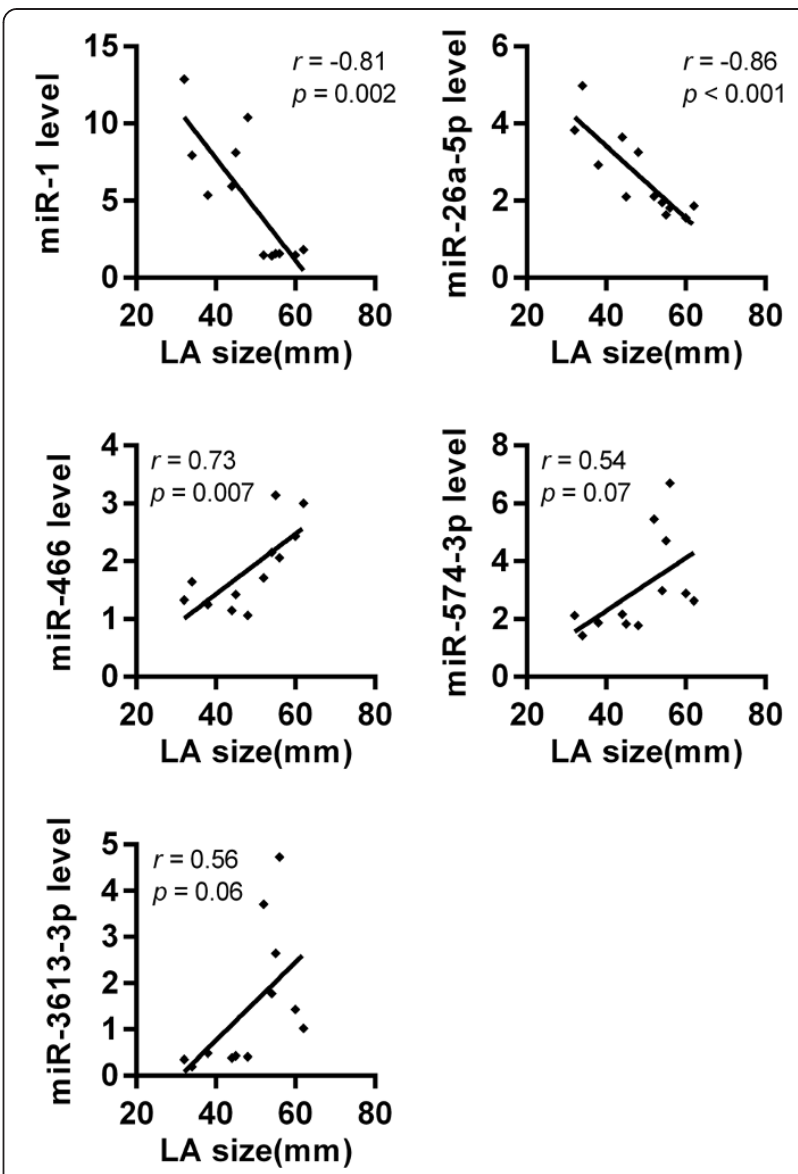

Figure 6 Correlations between LA size and the relative expression levels of selected miRNAs. Spearman's correlation analysis showed a positive correlation between the level of expression of miR-466 and LA size, and a negative correlation between the level of expression of miR-1 and miR-26a-5p with LA size. The expression levels of miR-574-3p and miR-3613-3p were not significantly correlated with LA size.

study is the first to create and compare miRNA profiles of the LA of MS patients with AF and those without AF. We found that in the LA of MS patients, 22 miRNAs were differentially expressed between those with AF and those in NSR.

The results of our study and that of Xiao et al. [16] were completely different, except for miR-26b. After eliminating the influences of the miRNA microarray technologies used in the two studies, we conclude that these differences, at least in part, may reflect different mechanisms involved in AF between the LA and RA. In MS patients, electrical remodeling of both the left and right atria [14] is intrinsic to the initiation, development, and maintenance of AF [25], and morphological differences have also been demonstrated between the two atria [26]. Thus, it is not surprising that AF alters the miRNA expression profiles of the LA of MS patients, and that these alterations may differ from those of the 
Table 3 Prediction of putative target genes and pathways of selected miRNAs

\begin{tabular}{lllll}
\hline miRNAs & Target gene & GO term & KEGG pathways & Target prediction database \\
\hline miR-1 & 613 & 41 & 2 & miRanda, MirTarget, microT, PicTar, TargetScan \\
miR-26a-5p & 591 & 16 & 2 & miRanda, MirTarget, microT, PicTar, TargetScan \\
miR-466 & 312 & 50 & 2 & MirTarget, TargetScan \\
miR-574-3p & 31 & 42 & 3 & MirTarget, microT, PicTar, TargetScan \\
miR-3613-3p & 847 & 133 & 14 & MirTarget, TargetScan \\
\hline
\end{tabular}

RA. These differences may reflect different mechanisms involved in AF between LA and RA. Therefore, investigations into the differences in miRNA expression profiles associated with AF in MS patients should focus not only on the RA but also on the LA.

Cooley et al. [17] investigated the differences in miRNA expression profiles in LAA tissues from valvular heart disease patients, and found no detectable differences between patients with AF and those with NSR, a lack that these researchers attributed partially to problems with tissue availability. However, Girmatsion et al. [27] reported that miR-
1 was downregulated in human LAA tissue from AF patients (relative to patients without $\mathrm{AF}$ who also underwent mitral valve repair or bypass grafting), which is consistent with our finding that miR-1 was downregulated in human LAA tissues in MS patients with AF. Unfortunately, Girmatsion et al.'s investigation did not utilize miRNA expression profiles. Recently, Luo et al. [28] found that miR-26 family members were significantly downregulated $(>50 \%)$ in LAAs from a canine AF model (miR-26a) and in right atrial appendages from AF patients (miR-26a and miR-26b). This suggests the possible involvement of these

Table 4 Biological processes of the predicted miRNA targets

\begin{tabular}{|c|c|c|}
\hline GO process & Target genes & $P$-value \\
\hline \multicolumn{3}{|l|}{ miR-1 } \\
\hline GO:0003723: RNA binding & DDX5 SFRS9 HNRNPU TRA2B CPEB1 SFRS1 HNRNPA3 HNRNPK SFRS3 & $6.57 \mathrm{E}-05$ \\
\hline GO:0006397: mRNA processing & $\begin{array}{l}\text { ISY1 DDX5 SFRS9 DHX15 HNRNPU CPEB1 SFRS1 HNRNPA3 } \\
\text { HNRNPK SFRS3 }\end{array}$ & $5.69 \mathrm{E}-03$ \\
\hline \multicolumn{3}{|l|}{ miR-26a-5p } \\
\hline $\begin{array}{l}\text { GO:0007179: transforming growth factor beta } \\
\text { receptor signaling pathway }\end{array}$ & TGFBR2 MAP3K1 NLK & $5.41 \mathrm{E}-04$ \\
\hline GO:0051301: cell division & CCNE2 CCNE1 CDK6 CCND2 & $7.90 \mathrm{E}-03$ \\
\hline \multicolumn{3}{|l|}{ miR-466 } \\
\hline GO:0003700: transcription factor activity & ARNT2 GLI3 SMAD2 RUNX1T1 TCF7L2 MECOM & $4.56 \mathrm{E}-04$ \\
\hline GO:0005515: protein binding & CCDC6 KIT CYCS EIF4E COL4A1 PTGS2 RUNX1T1 ULK2 MECOM RICTOR & $6.19 \mathrm{E}-04$ \\
\hline GO:0006468: protein amino acid phosphorylation & PRKAA2 RPS6KA3 SMAD2 ULK2 DAPK1 & $9.15 \mathrm{E}-04$ \\
\hline GO:0046872: metal ion binding & CYCS GLI3 PTGS2 RUNX1T1 MECOM & $6.58 \mathrm{E}-03$ \\
\hline \multicolumn{3}{|l|}{ miR-574-3p } \\
\hline GO:0031625: ubiquitin protein ligase binding & CUL2 ACVR1B & $1.46 \mathrm{E}-03$ \\
\hline GO:0051384: response to glucocorticoid stimulus & EP300 RXRA & $3.87 \mathrm{E}-03$ \\
\hline \multicolumn{3}{|l|}{ miR-3613-3p } \\
\hline GO:0003700: transcription factor activity & $\begin{array}{l}\text { NFATC3 NFAT5 EP300 RARA CREBBP RUNX1T1 RUNX1 ETS1 TCF7L2 } \\
\text { NKX3-1 HDAC2 STAT3 CTNNB1 SMAD4 }\end{array}$ & $5.59 \mathrm{E}-13$ \\
\hline GO:0004842: ubiquitin-protein ligase activity & $\begin{array}{l}\text { UBE2J1 UBE2H UBE2Z BIRC6 MAP3K1 UBE2N UBE2D3 UBE2B } \\
\text { NEDD4L LMO7 UBE2R2 HUWE1 UBE2E2 UBE2G1 }\end{array}$ & $5.61 \mathrm{E}-06$ \\
\hline GO:0006355: regulation of transcription, DNA dependent & NFAT5 EP300 RARA CREBBP ABL1 RUNX1T1 RUNX1 NKX3-1 CREB1 & $1.65 \mathrm{E}-03$ \\
\hline GO:0008134: transcription factor binding & PPARGC1A ETS1 TCF7L2 NKX3-1 HDAC2 PIM1 STAT3 CTNNB1 & $6.19 \mathrm{E}-06$ \\
\hline GO:0008284: positive regulation of cell proliferation & FGFR2 FGFR1 ADAM17 INSR KIT BIRC6 HDAC2 IGF1R CCND1 KRAS & $8.74 \mathrm{E}-04$ \\
\hline GO:0046872: metal ion binding & $\begin{array}{l}\text { PRKCI EP300 ACACB RARA CREBBP RUNX1T1 MID1 ADAM17 } \\
\text { INSR PPP1CB } \\
\text { PIM1 XIAP EGLN1 IGF1R LMO7 TRAF3 }\end{array}$ & 4.16E-04 \\
\hline GO:0051246: regulation of protein metabolic process & $\begin{array}{l}\text { UBE2J1 UBE2H UBE2Z BIRC6 UBE2N UBE2D3 UBE2B UBE2R2 } \\
\text { UBE2E2 UBE2G1 }\end{array}$ & $5.24 \mathrm{E}-05$ \\
\hline
\end{tabular}


Table 5 Pathway analysis of the selected miRNAs

\begin{tabular}{|c|c|c|}
\hline KEGG_Description & Target genes & $P$-value \\
\hline \multicolumn{3}{|l|}{ miR-1 } \\
\hline hsa3040: Spliceosome & ISY1 DDX5 SFRS9 DHX15 HNRNPU TRA2B SFRS1 HNRNPA3 HNRNPK SFRS3 & $1.39 \mathrm{E}-03$ \\
\hline hsa4320: Dorso-ventral axis formation & NOTCH2 EGFR NOTCH3 CPEB1 ETS1 & 3.86E-03 \\
\hline \multicolumn{3}{|l|}{ miR-26a-5p } \\
\hline hsa4115: p53 signaling pathway & CCNE2 CCNE1 PTEN CDK6 CCND2 IGF1 ATM & $1.98 \mathrm{E}-03$ \\
\hline hsa4010: MAPK signaling pathway & TGFBR2 MYC PPP3R1 CACNA1C PPP3CB MAP3K1 NLK PAK2 & $8.40 \mathrm{E}-03$ \\
\hline \multicolumn{3}{|l|}{ miR-466 } \\
\hline hsa4150: mTOR signaling pathway & PRKAA2 RPS6KA3 EIF4E ULK2 RICTOR & $2.36 \mathrm{E}-03$ \\
\hline hsa5200: Pathways in cancer & $\begin{array}{l}\text { CCDC6 ARNT2 KIT CYCS COL4A1 GLI3 PTGS2 SMAD2 RUNX1T1 TGFB2 } \\
\text { TCF7L2 DAPK1 MECOM }\end{array}$ & $4.56 \mathrm{E}-03$ \\
\hline \multicolumn{3}{|l|}{$\operatorname{miR}-574-3 p$} \\
\hline hsa5200: Pathways in cancer & CUL2 EP300 ACVR1B RXRA & $3.75 \mathrm{E}-03$ \\
\hline hsa5016: Huntington's disease & EP300 CLTC & $4.38 \mathrm{E}-03$ \\
\hline hsa5211: Renal cell carcinoma & CUL2 EP300 & $9.33 \mathrm{E}-03$ \\
\hline \multicolumn{3}{|l|}{ miR-3613-3p } \\
\hline hsa4520: Adherens junction & $\begin{array}{l}\text { TCF7 EP300 CREBBP ACTN4 FGFR1 PTPRJ INSR TCF7L2 ACVR1C IGF1R CTNNB1 } \\
\text { CTNND1 WASL LMO7 SMAD4 }\end{array}$ & $5.19 \mathrm{E}-06$ \\
\hline hsa4310: Wnt signaling pathway & $\begin{array}{l}\text { NFATC3 TCF7 NFAT5 EP300 CREBBP AXIN2 GSK3B PPP3CA VANGL2 TCF7L2 PPP2R5A } \\
\text { WNT5A CCND2 PPP3R1 CCND1 CTNNB1 CSNK1A1 CSNK1E WNT4 SMAD4 }\end{array}$ & $9.31 \mathrm{E}-05$ \\
\hline hsa4720: Long-term potentiation & EP300 CREBBP PPP1R12A PPP3CA PPP1CB PPP3R1 RPS6KA2 RAP1A GRIA1 KRAS & $6.75 \mathrm{E}-04$ \\
\hline hsa4330: Notch signaling pathway & JAG1 EP300 MAML3 CREBBP ADAM17 DLL1 NUMBL HDAC2 & $2.88 \mathrm{E}-03$ \\
\hline hsa4910: Insulin signaling pathway & PRKCI PPP1R3B PPARGC1A ACACB GSK3B PRKAA1 INSR SOCS3 IRS1 EIF4E PPP1CB KRAS IRS2 & $6.80 \mathrm{E}-03$ \\
\hline hsa4920: Adipocytokine signaling pathway & PPARGC1A ACACB PRKAA1 SOCS3 IRS1 STAT3 ACSL4 ACSL6 IRS2 & $8.20 \mathrm{E}-03$ \\
\hline
\end{tabular}

miRNAs in AF pathophysiology, and is consistent with our finding that miR-26a-5p was downregulated in LAA tissues from MS patients with AF compared with those who remained in NSR.

$\mathrm{Lu}$ et al. [10] found that levels of miR-328 were elevated 3.5- to 3.9-fold in LAAs from dogs with model AF and in right atrial appendages from AF patients, detected by both microarray and RT-qPCR. However, in the present study we found that the expressions of miR328 were very low in both the NSR and AF group and were not significantly different between the two groups. This contradictory finding may reflect differences in the species (dogs in Lu et al., humans in the present study) and in tissues that were sampled (right atrial appendages from AF patients in Lu et al., LAAs from AF patients in the current study) and the heterogeneity of human myocardial samples [29].

Studies have shown that miRNAs may be involved directly or indirectly in AF by modulating atrial electrical remodeling (miR-1, miR-26, miR-328) [10,27,28] or structural remodeling (miR-30, miR-133, mir-590) $[7,30]$. One study showed that miR-1 overexpression slowed conduction and depolarized the cytoplasmic membrane by post-transcriptionally repressing $K C N J 2$ (potassium inwardly-rectifying channel, subfamily J, member 2; which encodes the $\mathrm{K}^{+}$channel subunit Kir2.1) and GJA1 (gap junction protein, alpha 1, $43 \mathrm{kDa}$; which encodes connexin 43), and this likely accounts at least in part for its arrhythmogenic potential [31]. Another study indicated that miR-1 levels are greatly reduced in human AF, possibly contributing to upregulation of Kir2.1 subunits, leading to increased cardiac inward-rectifier potassium current $I_{K 1}$ [27]. A recent study identified miR-26 as a potentially important regulator of $K C N J 2$ gene expression and, via $\mathrm{I}_{\mathrm{K} 1}$, a determinant of AF susceptibility [28]. In addition, it also identified miR-26 as a potential mediator of the electrophysiological effects of $\mathrm{Ca}^{2+}$-dependent NFAT (nuclear factor of activated $\mathrm{T}$ cells) signaling, believed to be important in the perpetuation of AF.

Previously, the miR-466, miR-574, and miR-3613 have not been described as participating in cardiovascular pathology. The current study found that these miRNAs are potentially involved in several important biological processes and functional pathways associated with AF (e.g., mTOR, Wnt, and Notch signaling), based on the predictions of putative target genes and pathways determined via miRFocus. Our results may implicate these miRNAs in the pathogenesis of AF. 
In MS, the association between LA size and AF is well established and LA dilatation is considered both a cause and consequence of AF [13]. Our study found that the expression levels of three validated miRNAs (miR-1, miR-26a-5p, miR-466) correlated with LA size, while those of two others (miR-574-3p, miR-3613-3p) did not. This discrepancy is probably due to the multifactorial nature of AF in MS. For example, it is likely that persistent rheumatic inflammation and LA fibrosis also contribute to the etiology of AF in MS, as well as LA size and hypertension [13].

The main limitation of this study was the small number of patients included. This was due, in part, to the difficulty of finding MS patients with NSR. In addition, because this study was performed with native human tissues, we could not conduct experiments to modulate miRNA levels. Accordingly, the evidence presented here is indirect. Furthermore, the exact targets and pathways by which alterations in miRNAs cause AF in MS patients remain elusive and deserve further investigation [16]. Finally, the patients in this study were a specific cohort with preserved systolic left ventricular function and little comorbidity; they were undergoing mitral valve replacement surgery. Thus, changes identified in this population may not be representative of other cohort populations [27].

\section{Conclusions}

This study shows that AF alters the miRNA expression profiles in LA from MS patients. These findings may be useful for the biological understanding of AF in MS patients and provide potential therapeutic targets for AF [32].

\section{Additional files}

Additional file 1: Raw signal values for all miRNAs in microarray.

Additional file 2: Primers for RT-qPCR of miRNA and RNU6B.

Additional file 3: RT-qPCR data.

\section{Abbreviations \\ AF: Atrial fibrillation; CV: Coefficient of variation; GO: Gene Ontology; KEGG: Kyoto Encyclopedia of Genes and Genomes; LA: Left atrial; LAA: Left atrial appendage; miRNA: Microrna; MS: Mitral stenosis; NSR: Normal sinus rhythm; NYHA: New York Heart Association; RT-qPCR: Reverse-transcription quantitative PCR; TGFBR: Transforming growth factor beta receptor.}

\section{Competing interests}

The authors declare that they have no competing interests.

\section{Authors' contributions}

$\mathrm{HL}$ performed the molecular studies, participated in the sequence alignment, and drafted the manuscript. GXC, MYL, HQ, JR, and JPY participated in open heart surgery and collected clinical samples. $\mathrm{HL}$ and ZKW participated in the design of the study and performed the statistical analyses. ZKW and GXC conceived the study, participated in its design and coordination, and helped to draft the manuscript. HL and GXC contributed equally to this article. All authors read and approved the final manuscript.

\section{Acknowledgements}

This research project was supported by the National Basic Research Program of China (973 Program; NO. 2010CB5295007), the Pearl River Scholar Program (80000-3210003), and the National Science Fund for Young Scholars (81000061).

We would like to acknowledge Gang Dai and Rong Fang (Key Laboratory on Assisted Circulation) for experimental assistance. We are grateful to Junming Yao of LC-Bio for technical assistance.

\section{Author details}

'Second Department of Cardiac Surgery, First Affiliated Hospital of Sun Yat-Sen University, 58 Zhongshan II Road, Guangzhou 510080, China.

2Department of Cardiopulmonary Bypass, First Affiliated Hospital of Sun Yat-Sen University, Guangzhou, China.

Received: 20 October 2013 Accepted: 22 January 2014

Published: 25 January 2014

\section{References}

1. Wolf PA, Abbott RD, Kannel WB: Atrial fibrillation: a major contributor to stroke in the elderly. The Framingham Study. Arch Intern Med 1987, 147(9):1561-1564.

2. Brugada R, Tapscott T, Czernuszewicz GZ, Marian AJ, Iglesias A, Mont L, Brugada J, Girona J, Domingo A, Bachinski LL, et al: Identification of a genetic locus for familial atrial fibrillation. N Engl J Med 1997, 336(13):905-911.

3. Nattel S: New ideas about atrial fibrillation 50 years on. Nature 2002, 415(6868):219-226.

4. Chen JF, Callis TE, Wang DZ: Micrornas and muscle disorders. J Cell Sci 2009, 122(Pt 1):13-20.

5. Cordes KR, Srivastava D, Ivey KN: MicroRNAs in cardiac development. Pediatr Cardiol 2010, 31(3):349-356.

6. Wang N, Zhou Z, Liao X, Zhang T: Role of microRNAs in cardiac hypertrophy and heart failure. IUBMB Life 2009, 61(6):566-571.

7. Shan H, Zhang Y, Lu Y, Pan Z, Cai B, Wang N, Li X, Feng T, Hong Y, Yang B: Downregulation of miR-133 and miR-590 contributes to nicotine-induced atrial remodelling in canines. Cardiovasc Res 2009, 83(3):465-472.

8. Shi B, Guo Y, Wang J, Gao W: Altered expression of microRNAs in the myocardium of rats with acute myocardial infarction. BMC CardiovasC Disord 2010, 10:11.

9. Qin $\mathrm{H}$, Chen GX, Liang MY, Rong J, Yao JP, Liu H, Wu ZK: The altered expression profile of microRNAs in cardiopulmonary bypass canine models and the effects of mir-499 on myocardial ischemic reperfusion injury. J Transl Med 2013, 11(1):154.

10. Lu Y, Zhang Y, Wang N, Pan Z, Gao X, Zhang F, Shan H, Luo X, Bai Y, Sun L, et al: MicroRNA-328 contributes to adverse electrical remodeling in atrial fibrillation. Circulation 2010, 122(23):2378-2387.

11. Dawson K, Wakili R, Ordog B, Clauss S, Chen Y, Iwasaki Y, Voigt N, Qi XY, Sinner MF, Dobrev D, et al: MicroRNA29: a mechanistic contributor and potential biomarker in atrial fibrillation. Circulation 2013, 127(14):1466-1475. 1475e1461-1428.

12. Camm AJ, Kirchhof P, Lip GY, Schotten U, Savelieva I, Ernst S, Van Gelder IC, Al-Attar N, Hindricks G, Prendergast B, et al: Guidelines for the management of atrial fibrillation: the Task Force for the Management of Atrial Fibrillation of the European Society of Cardiology (ESC). Eur Heart J 2010, 31(19):2369-2429.

13. Carabello BA: Modern management of mitral stenosis. Circulation 2005, 112(3):432-437.

14. John B, Stiles MK, Kuklik P, Chandy ST, Young GD, Mackenzie L, Szumowski L, Joseph G, Jose J, Worthley SG, et al: Electrical remodelling of the left and right atria due to rheumatic mitral stenosis. Eur Heart J 2008, 29(18):2234-2243.

15. Marin F, Roldan V, Monmeneu JV, Bodi V, Fernandez C, de Burgos FG, Marco P, Sogorb F: Prothrombotic state and elevated levels of plasminogen activator inhibitor-1 in mitral stenosis with and without atrial fibrillation. Am J Cardiol 1999, 84(7):864. A9.

16. Xiao J, Liang D, Zhang Y, Liu Y, Zhang H, Li L, Liang X, Sun Y, Chen YH: MicroRNA expression signature in atrial fibrillation with mitral stenosis. Physiol Genomics 2011, 43(11):655-664.

17. Cooley N, Cowley MJ, Lin RC, Marasco S, Wong C, Kaye DM, Dart AM, Woodcock EA: Influence of atrial fibrillation on microRNA expression profiles in left and right atria from patients with valvular heart disease. Physiol Genomics 2012, 44(3):211-219. 
18. Huang WJ, Zhou R, Zeng XR, Tan XQ, Cheng ZH, Tang MH, Gou LT, Chen LJ, Tong AP, He Y, et al: Comparative proteomic analysis of atrial appendages from rheumatic heart disease patients with sinus rhythm and atrial fibrillation. Mol Med Report 2011, 4(4):655-661.

19. Shah MY, Pan X, Fix LN, Farwell MA, Zhang B: 5-Fluorouracil drug alters the microRNA expression profiles in MCF-7 breast cancer cells. J Cell Physiol 2011, 226(7):1868-1878.

20. Bolstad BM, Irizarry RA, Astrand M, Speed TP: A comparison of normalization methods for high density oligonucleotide array data based on variance and bias. Bioinformatics 2003, 19(2):185-193.

21. Boggs RM, Wright ZM, Stickney MJ, Porter WW, Murphy KE: MicroRNA expression in canine mammary cancer. Mamm Genome 2008, 19(7-8):561-569.

22. Mortarino M, Gioia G, Gelain ME, Albonico F, Roccabianca P, Ferri E, Comazzi S: Identification of suitable endogenous controls and differentially expressed microRNAs in canine fresh-frozen and FFPE lymphoma samples. Leuk Res 2010, 34(8):1070-1077.

23. Zeiger U, Khurana TS: Distinctive Patterns of MicroRNA Expression in Extraocular Muscles. Physiol Genomics 2010, 41(3):289-296.

24. Modrego J, Maroto L, Tamargo J, Azcona L, Mateos-Caceres P, Segura A, Moreno-Herrero R, Perez-Castellanos N, Delpon E, Perez-Villacastin J, et al: Comparative Expression of Proteins in Left and Right Atrial Appendages From Patients With Mitral Valve Disease at Sinus Rhythm and Atrial Fibrillation. J Cardiovasc Electrophysiol 2010, 21(8):859-868.

25. Sharma $D, L i G, X u G, L i u$ Y, Xu Y: Atrial remodeling in atrial fibrillation and some related microRNAs. Cardiology 2011, 120(2):111-121.

26. Chapeau C, Gutkowska J, Schiller PW, Milne RW, Thibault G, Garcia R, Genest $J$, Cantin M: Localization of immunoreactive synthetic atrial natriuretic factor (ANF) in the heart of various animal species. J Histochem Cytochem 1985, 33(6):541-550.

27. Girmatsion Z, Biliczki P, Bonauer A, Wimmer-Greinecker G, Scherer M, Moritz A, Bukowska A, Goette A, Nattel S, Hohnloser SH, et al: Changes in microRNA-1 expression and IK1 up-regulation in human atrial fibrillation. Heart Rhythm 2009, 6(12):1802-1809.

28. Luo X, Pan Z, Shan H, Xiao J, Sun X, Wang N, Lin H, Xiao L, Maguy A, Qi XY, et al: MicroRNA-26 governs profibrillatory inward-rectifier potassium current changes in atrial fibrillation. J Clin Invest 2013, 123(5):1939-1951.

29. Ikeda S, Kong SW, Lu J, Bisping E, Zhang H, Allen PD, Golub TR, Pieske B, Pu WT: Altered microRNA expression in human heart disease. Physiol Genomics 2007, 31(3):367-373.

30. Li H, Li S, Yu B, Liu S: Expression of miR-133 and miR-30 in chronic atrial fibrillation in canines. Mol Med Report 2012, 5(6):1457-1460.

31. Yang B, Lin H, Xiao J, Lu Y, Luo X, Li B, Zhang Y, Xu C, Bai Y, Wang H, et al: The muscle-specific microRNA miR-1 regulates cardiac arrhythmogenic potential by targeting GJA1 and KCNJ2. Nat Med 2007, 13(4):486-491.

32. Condorelli G, Latronico MV, Dorn GWN: MicroRNAs in heart disease: putative novel therapeutic targets? Eur Heart J 2010, 31(6):649-658.

doi:10.1186/1471-2261-14-10

Cite this article as: Liu et al:: Atrial fibrillation alters the microRNA expression profiles of the left atria of patients with mitral stenosis. BMC Cardiovascular Disorders 2014 14:10.

\section{Submit your next manuscript to BioMed Central and take full advantage of:}

- Convenient online submission

- Thorough peer review

- No space constraints or color figure charges

- Immediate publication on acceptance

- Inclusion in PubMed, CAS, Scopus and Google Scholar

- Research which is freely available for redistribution

Submit your manuscript at www.biomedcentral.com/submit
Biomed Central 